\title{
Téoros
}

Revue de recherche en tourisme

\section{La mise en valeur muséologique et touristique du village d'Hébertville}

\author{
Une approche muséale nouvelle pour une galerie d'art ouverte
}

Jean-Claude Jay-Rayon et Brigitte Morneau

Volume 11, numéro 2, juillet 1992

Quand les musées s’ouvrent au tourisme...

URI : https://id.erudit.org/iderudit/1078090ar

DOI : https://doi.org/10.7202/1078090ar

Aller au sommaire du numéro

Éditeur(s)

Université du Québec à Montréal

ISSN

0712-8657 (imprimé)

1923-2705 (numérique)

Découvrir la revue

Citer cet article

Jay-Rayon, J.-C. \& Morneau, B. (1992). La mise en valeur muséologique et touristique du village d'Hébertville : une approche muséale nouvelle pour une galerie d'art ouverte. Téoros, 11(2), 34-38. https://doi.org/10.7202/1078090ar 


\section{La mise en valeur muséologique et touristique du village d'Hébertville}

Une approche muséale nouvelle pour une galerie d'art ouverte Jean-Claude Jay-Rayon, en collaboration avec Brigitte Morneau*

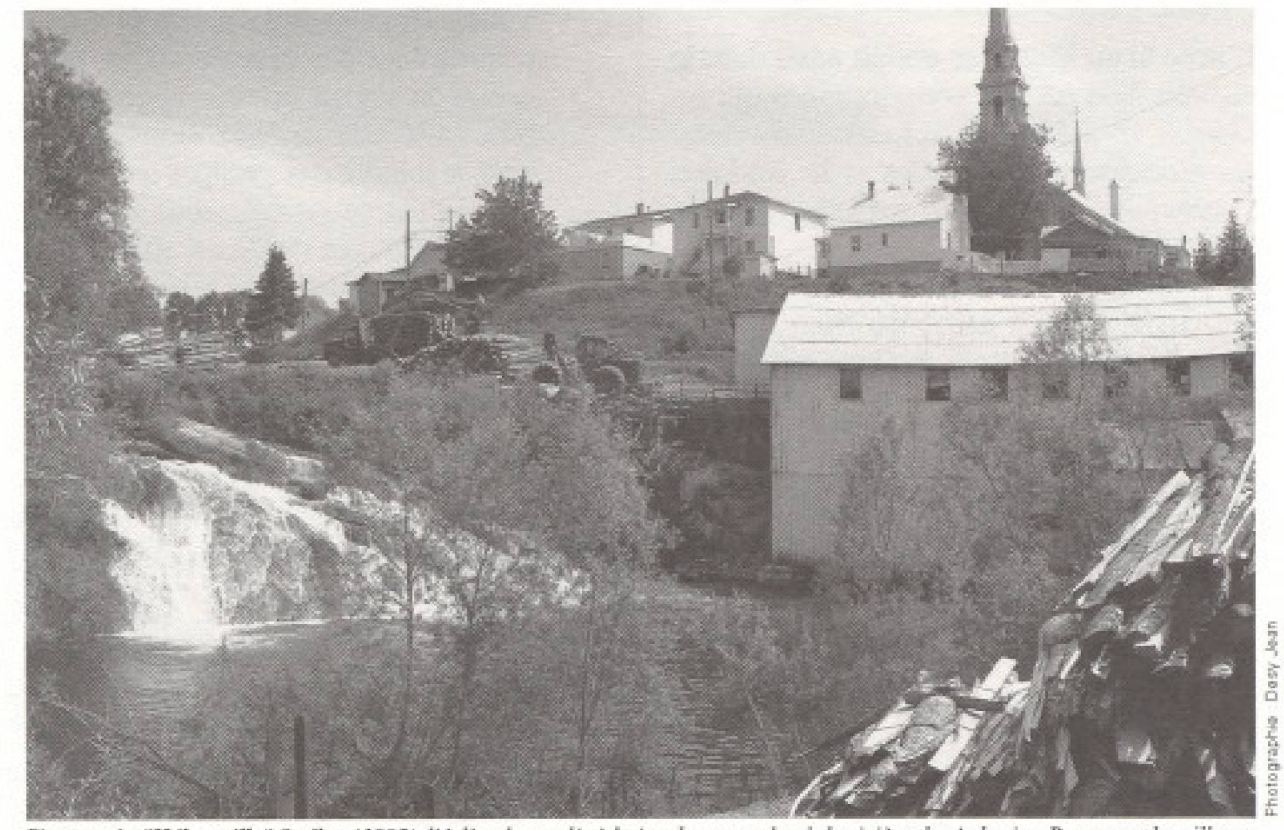

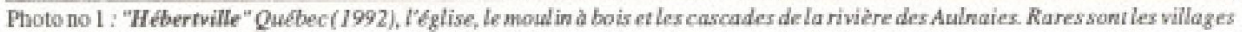

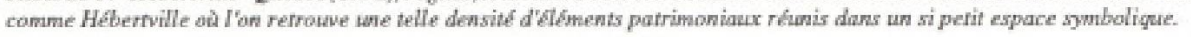

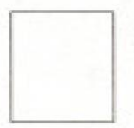

Le village d'Hébertville, situé à l'entrée du lac Pikouagami - lac plat - communément appelé Lac Saint-Jean - pourrait rapidement devenir, en réalisant son projet muséal original, une excursion recherchée par laclientèle touristique de la ville de Québec. Principale porte de la région et située le long de la route 169, cette municipalité bénéficie déjà d'une circulation touristique proxémique de 25000 personines ${ }^{(1)}$.

Ainsi, cette petite agglomération de 2500 personnes environ a l'intention de construire un type de développement local à la fois muséalet touristique d'un tout nouveaugenre. Son idée étant de représenter des faits et gestes reliés à son passé sur de grandes murales peintes et de placer ces dernières sur certains murs, de façonà constituer un circuit patrimonial digne d'intếrêt.

Une telle mise en valeur à la fois culturelle, touristique et économique n'ayant pas encore été réalisće au Québec, le projet se situe à l'avant-garde, parce qu'il veut faire ressortir l'identité du village par une galerie d'art populaire, ouverte comme une fenêtre sur le patrimoine vivant ancestral.
Quant au succès escompté au plan de la rentabilité et du tourisme, ce dernier fait peu de doute si on le compare à des réalisations similaires déjà en fonction au Canada ou en Europe.

Hébertville.:. paroisse fondatrice carrefour authentique et symbolique facile à sacraliser ${ }^{[2]}$

Le village d'Hébertville nommé ainsi en l'honneur du curé Nicolas Tolentin Hébert, fut fondé en 1849 - 202 ans après que le Père Jean Dequen, en 1647, fut lui-même passé à cet endroiten allant porter religionet secours à des amérindiens malades.

Ce site a donc été retenu parce qu'il représente à la fois un gisement et une sorte de cle environnementale ${ }^{(3)}$ qui contrôle $21 \%$ de la circulation touristique de la région concernée. Il est ainsi stratćgiquement bien situé au sein de l'histoire et de la géographie régionale, pouvant devenir un symbole fort, tout en conservant son authenticité. Ce que le touriste exige de plus en plus! 
En réalité, il ne s'agit pas d'une paroissemère quelconque, mais d'un endroit de transition significatif sur la route des fourrures, d'un haut lieu de mission et de colonisation, de défricheurs, de cultivateurs, de petites industries, de marchés et commerces. Le tout, contenu dans un espace relativement restreint, apte à générer un produit culturel et touristique à haute densité. (voir photo no 1)

Ce dernier élément est majeur car le touriste exige, lors de ses voyages, d'obtenir un maximum de produits culturels et naturels dans un minimum d'espace et de temps. Efficacité oblige! Enérigeantdonc, aucentre même du village - dans le carré patrimonial - une vingtaine de murales évocatrices du passé, le produit ainsi élaboré sera intéressant et acquerra des capacités de rentabilité, parce que concentré en un seul endroit.

Il en serait par contre tout autrement si l'on pratiquait un type d'écomuséologie disperséeet sil'on exigeait du touriste qu'il sillonne toute la région pour obtenir le même résultat!

\section{Le coeur du projet muséal... une galerie d'art populaire, facile de lecture et d'accès}

Une certaine expérience dans la mise en valeur touristique du patrimoine maritime, agraire, industriel ou primitif au cour's des cinq dernières années, nous permet, du moins en ce qui concerne les régions ressources, de dégager une constante majeure. Les gens qu'il s'agisse de visiteurs ou de visités, réagissent positivement et $s^{\prime}$ impliquent facilement à partir de certaines représentations culturelles. Mais, pas à partir de toutes, ou de n'importe lesquelles et c'est ce qu'il est important de bien saisir!

Les gens se perçoivent très concernés lorsque l'on fait référence au passé réeldes aieux et en particulier à leur manière de vivre et donc... d'avoir été! Le tout appuye par des anecdotes, d'anciennes photos, dessins, légendes... qui relatent des gestes coutumiers ou des événements tels que les noces, les processions, les marchés, la première voiture dans la rue, les fenaisons et moissons, les incendies, etc.

En un certain sens, nous devons comprendre que les cultures locales sont enracinées dans une terre concrète où l'imaginaire est presque toujours figuratif et naîf ${ }^{(4)}$, quoique souvent enjolivé. À l'opposé, le touriste, lui, souhaite de préférence aborder ces mêmes localités dans une recherche du passé-présent. Il y a donc ainsi quelque part, un point de rencontre souhaitable qu'il s'agit de concrétiser avec justesse et qualité et ce, en favorisant une approche qui se réfere surtout à l'écologie humaine.

Dans le cas d'Hébertville, quatre périodes ont êtế retenues pour être picturalement réalisées:

- avant 1849 vers 1647 ... pour ce qui concerne les tableaux retraçant la vie amérindienne, celles des coureurs des bois, des missionnaires et des commerçants de fourrures;

- $1849 \ldots$ pour les tableaux relatant la fondation et la construction du village ainsi que le défrichage:

- 1850-60 et plus ... pour les scènes bucoliques rattachées à la vie agraire, familiale et religieuse de la fin du XIX ${ }^{e}$ siècle;

- 1870-1900 et plus ... pour les macropeintures se rapportant aux moulins à farine et à bois, aux marchés et commerces généraux, ainsi qu'à la pratique professionnelle de la petite bourgeoisie naissante.

Enlin, si le projet muséal aété orienté de cette façon par le maître d'oeuvre et le concepteur, c'est que, comme le précisent MarcLaplante et Louise Trottier, *... le touriste est trè̀s friand d' histoire, du passé, des origines, des fondations [...] les vieilles choses lui paraissent plus authentiques ${ }^{(5)}$.

\section{Éléments référentiels... en vue d'une meilleure compréhension}

Quoique tout le projet aitété conçu pourcréer une attraction unique au niveau local et régional, $\infty$ dernier repose sur une série de principes fondamentaux encore peu utilisés en amenagement touristique. Et comme ceux-ci ne se réferent qu'indirectement à la sociologie, il est nćcessaire de mentionner qu'ilsoriginent principalementdel'éthologie humaine ${ }^{(6)}$, de la proxémique $e^{(7)}$, de l'écologie humaine, de la peroeption du mouvement, des formes, textures, couleurs, etc. En tout, le projetd'Hébertville anécessité l'utilisation d'environ 500 facteurs référentiels déterminants.
Par exemple, le choix de représenter le passé humain du village sur des peintures monumentales extérieures est basé sur le principe que la personne retiendrait instantanément dans n'importe quel environnement ce qui est le plus volumineux, inusité et... anthropomorphique.

Ce qui est macro serait alors décodé comme naturellement plus performant au-delà du champ conscient des visiteurs et aussi des visités. Dans le cas d'Hébertville, le passé humain invisible, parce que mort et disparu, se verra donc maximisé et concrètement agrandi. Il reprendra forme comme patrimoine vivant surdimensionné et constituera alors un attrait épique que le touriste ne pourra perceptuellement esquiver.

Comme on le constate, il ne suffit plus d' avoir un passé contenu dans les livres ou sur des photos jaunies pour que celui-ci capte l'attention du touriste et en conséquence fasse d'un lieu, une destination. Il s'agit dorénavant d'ajouter une valeur: celle du grandiose! Il en est đe même de la réfection isolée d'un édifice patrimonial, ouencore, deladispersion du patrimoine immobilier dans une région: l'une et l'autre seront peu efficaces au plan touristique parce qu'éparpillées dans un espace trop grand.

Un deuxième exemple permettra de saisir encore davantage la démarche sous-jacente. Il a êté prévu, pour que le touriste puisse mieux vivre le passé, que l'on aménage au sein même des peintures, des éléments en trompe-l'oeil. Cette technique ayant pour but de créer des images et des leurres dont il est difficile pour le cerveau de discemer le vrai du faux. Ainsi, on envisagera de faire entrer le visiteur dans l'histoire par l'illusion d'optique tout en l'amusant sensoriellement.

Lors de nosobservations directes, nous avons d'ailleurs pu nous rendre compte que des peintures figurant des scènes d'antan étaient à ce point performantes qu'elles amenaient letouristeà...jouer avec le contenu del'image. D'où la force évocatrice de cette approche interactive par rapport à une autre!

Enfin, à tout cela s"ajoutera l'utilisation des formes arrondies pour les paysages et les personnages, des couleurs vives pour les vêtements, des attitudes, un climat paisible concernant les scènes bucoliques, etc. Le tout créant un décor capable de susciter des émotions précises. 


\section{Problématiques et contraintes... passées et à venir!}

Dans l'ensemble, le projet a franchi avec succès, en deux ans, les principales étapes d'un démarrage réussi, soit: l'acceptation du milieu, par résolutions, de privilégier ce type particulier de mise en valeur patrimoniale, uneétude conceptuelle ${ }^{(3)}$ etcommerciale $\mathrm{e}^{(9)}$, un plan de formation de 600 heures ${ }^{(10)}$ et la mise sur pied d'une agence réceptive ${ }^{(11)}$.

Malgré tout, ces réalisations ne peuvent escamotercertains problèmes réels qu'il s'agira de résoudre au fur et à mesure. Une problématique qui, à bien des égards, est similaire a celle qui touche le monde municipal en cette période de difficile transition et de mutation du développement régional. C'est pourquoi nous croyonsutile de nous y attarder un peu!

Le villaged'Hébertville,comme biend'autres avec lui un peu partout, avait misé depuis une quinzaine d'années sur une démarche de loisir subventionné de type plein-air et socioculturel, régional, la confondant, involontairement sans doute, avec un tourisme industriel et international. Le bilan chiffré de ces choix est à notre avis éclairant!

La municipalité a gếré une dépense đ'environ $87000 \$$ en études pour des recherches d'idées et de rentabilité. Elle a emprunté 600 000 \$a sa caisse populaire et fait investir 225 sociétaires privés pour $225000 \$$. Elle aaussi reçu $300000 \$$ en publicité et a été subventionnée pour 1.9 million $\$$ et 273000 \$ en 1991. Elle a aussi investi elle-même sur cinq ans, $983000 \$$ \$(12).

Tout ceci (le centre de ski, le thêâtre d'été, l'érablière, etc., la venue du Cirque du tonnerre, qui devait en principe créer un événement rentable), a abouti à des déficits de 40000 \$ en 1990, de 260000 \$en 1991 et de $160000 \$$ en 1992 !

Une telle situation, dans certaines petites localités, n'est pas rare de nos jours et elle pose, au-delà d' un bilan seulement déficitaire, le problème de l'épuisement des investissements régionaux et de la nécessitê, dorénavant, de faire des choix qui généreront des profits et des emplois directs. C'est dans ce contexte que le projet muséal d'Hébertville se situe actuellement, devant recréer une confiance et trouver les 300000 \$ nécessaires à l'érection des murales.

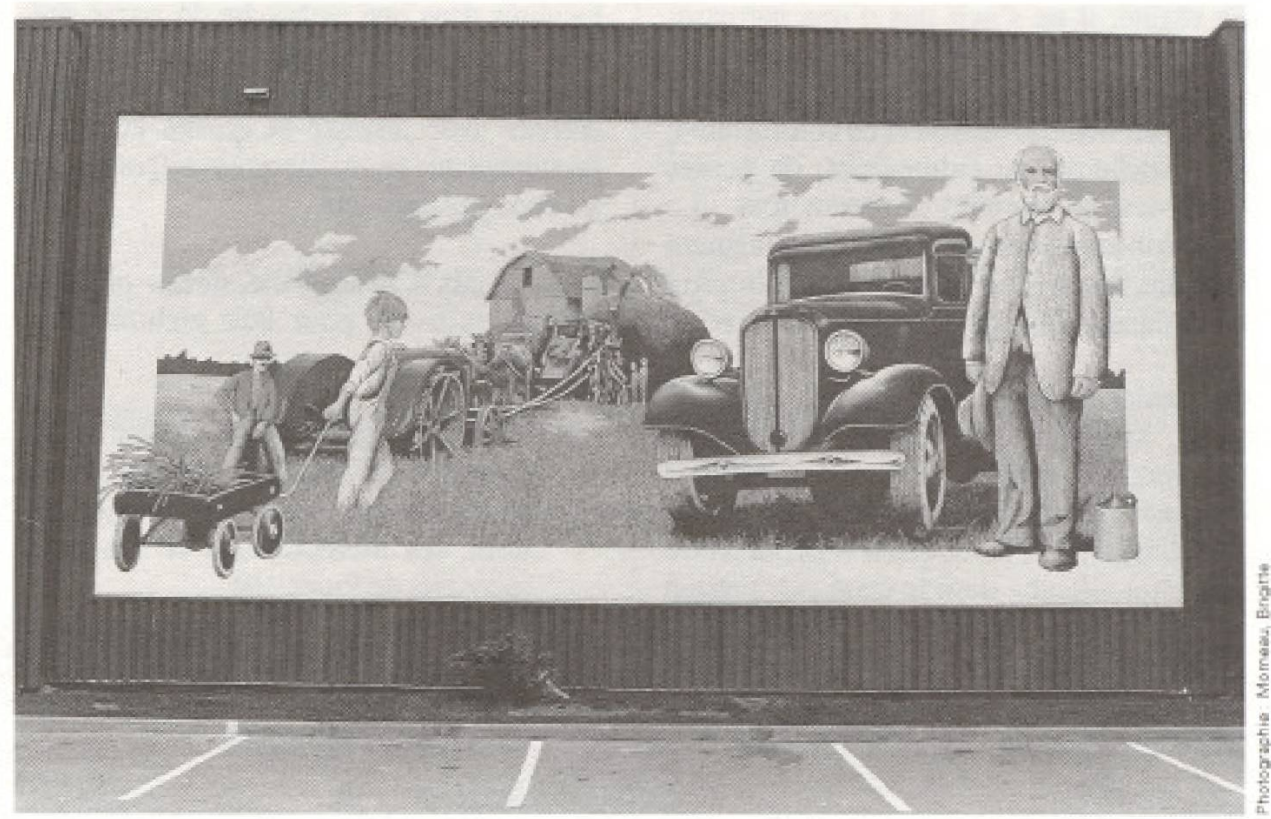

Photo no 2 : "LTTLE HELPER" (1989), par Dan Sawatzky, Chemainus, C. B..

Cetfe wurafe inposante de 40' $X 18^{\prime}$ met l'enphuse symbolique sur une certaine périoule agraire et le mode de vie qui en découlait

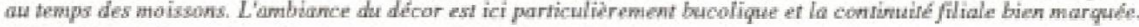

Le deuxième constat est d'ordre spatial et concerne la qualité de l'environnement urbain. En investissant à plus de 10 kilomètres dans le centre de plein-air, on a en quelque sorte, abandonné temporairement le carré patrimonial et la rivière des Aulnaies dans le village même. Exactement là où les touristes et les visités souhaitent le plus se rencontrer!

Il s'agit donc maintenant tout à la fois de dépolluer le cours d'eau, de nettoyer ses berges et de remettre en valeur le patrimoine immobilier. En d'autres termes, il s'agit de recentrer le développement, d'améliorer la qualité de vie des habitants et de créer l'attraction culturelle dominante (les murales) pour les visiteurs.

La troisième contrainte détectée, elle, se situe à un niveau plus subtil mais combien souvent présente quand il s'agit d'innover! Ellereleve, à la fois, d' une certaine orthodoxie en matière culturelle, des goûts personnels, voire d'une certaine idéologie et elle interpelle directement la création artistique et la libertê d'expression qui doit l'entourer.

En ce sens, la nouvelle politique en matière culturelle précise que, wl'État n'a pas à faire de choix entre les différentes tendances artistiques [...]. Par ailleurs, les conditions rattachées à l'octroi du soutien, qu'il soit technique ou financier, doivent être exempts de toute ingérences ${ }^{(13)}$.
Cette toute récente garanlie gouvernementale est d'autant plus importante pour le village d'Hêbertville que l'originalité de la démarche muséale, pourtant fondée sur des critères universels, semble déjà souffrir d'un manque de compréhension et d'ouverture. Ainsi, sans doute, enest-il du sort réservé aux projets qui se situent hors des nomes. Phénomènes courants soit, mais qu'il faudra un jour surpasser si certaines régions souhaitent parfaire une identité culturelle et atteindre une capacité de mise en marché touristique d'envergure.

\section{Prospectives... sur un fond de réflexion concrète}

Que l'on en soit peu ou pas convaincu, les inventaires patrimoniaux complets de chaque ville et village du Québec, sans mise en valeur touristique spectaculaire, n'aboutiront au mieux qu'à augmenter la masse d'information disponible, sans pour autant susciter l'intếrêt général d'une clientèleen vacances.

À cela s'ajoute que le fait de bâtir partout des circuits patrimoniaux similaires et parfois intellectuellement fastidieux d'accès, risque peu d'influencer en profondeur l'industrie culturelle et touristique associées.

C'est ce que le village d'Hébertville a tenté d'éviter en créant un produit unique, parce que monumental, qui devra être capable $\alpha . .$. 


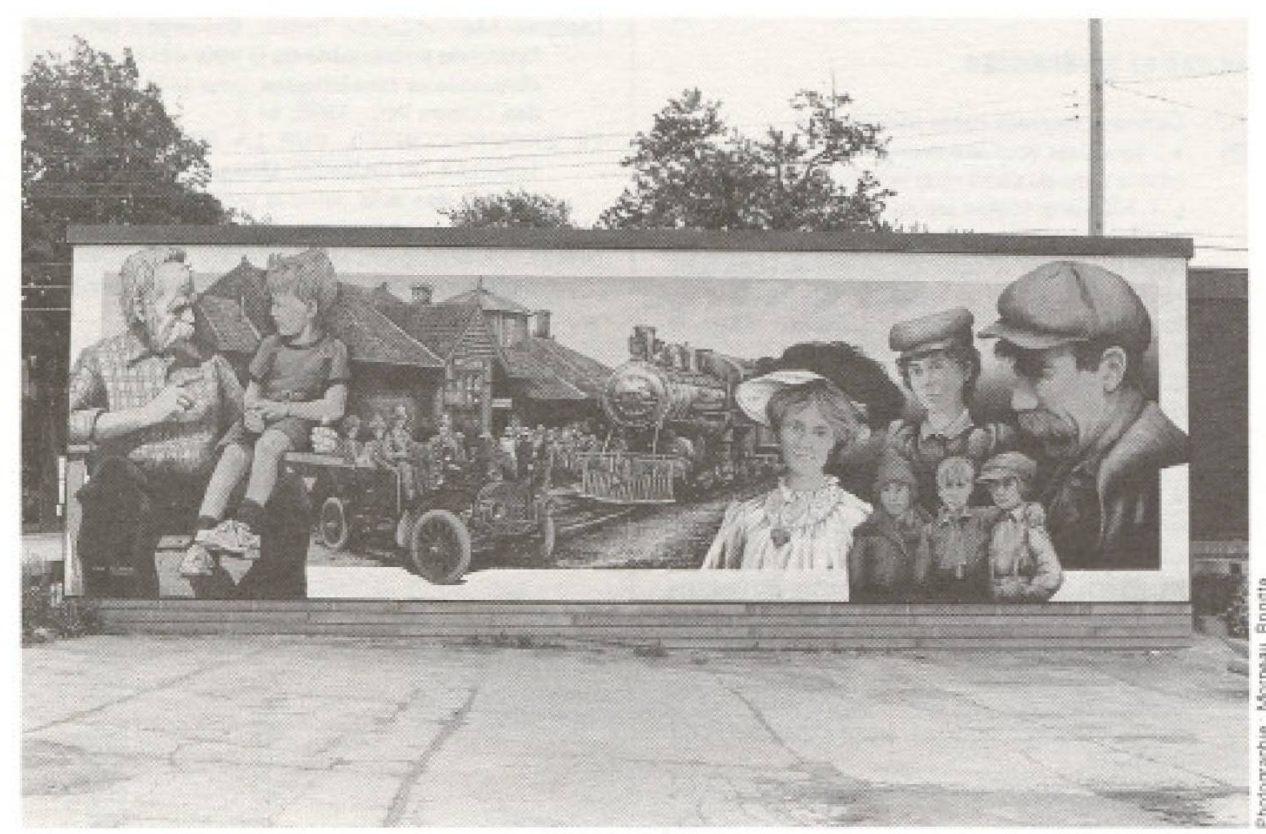

Photo no 3: "TELL ME ABOUT THE OLDEN DAYS" (1988), par Dan Sawatzky, Chemainus, C B.

Cerlé murale comprosée qui mesure $50^{\prime} X 20^{\circ}$ a éé peinte en encourageant des enjants de l'endroí à participer à sa ralisafian et ce, de façon à créer un lien concret entre le touriste, le visité et le produit touristigue.

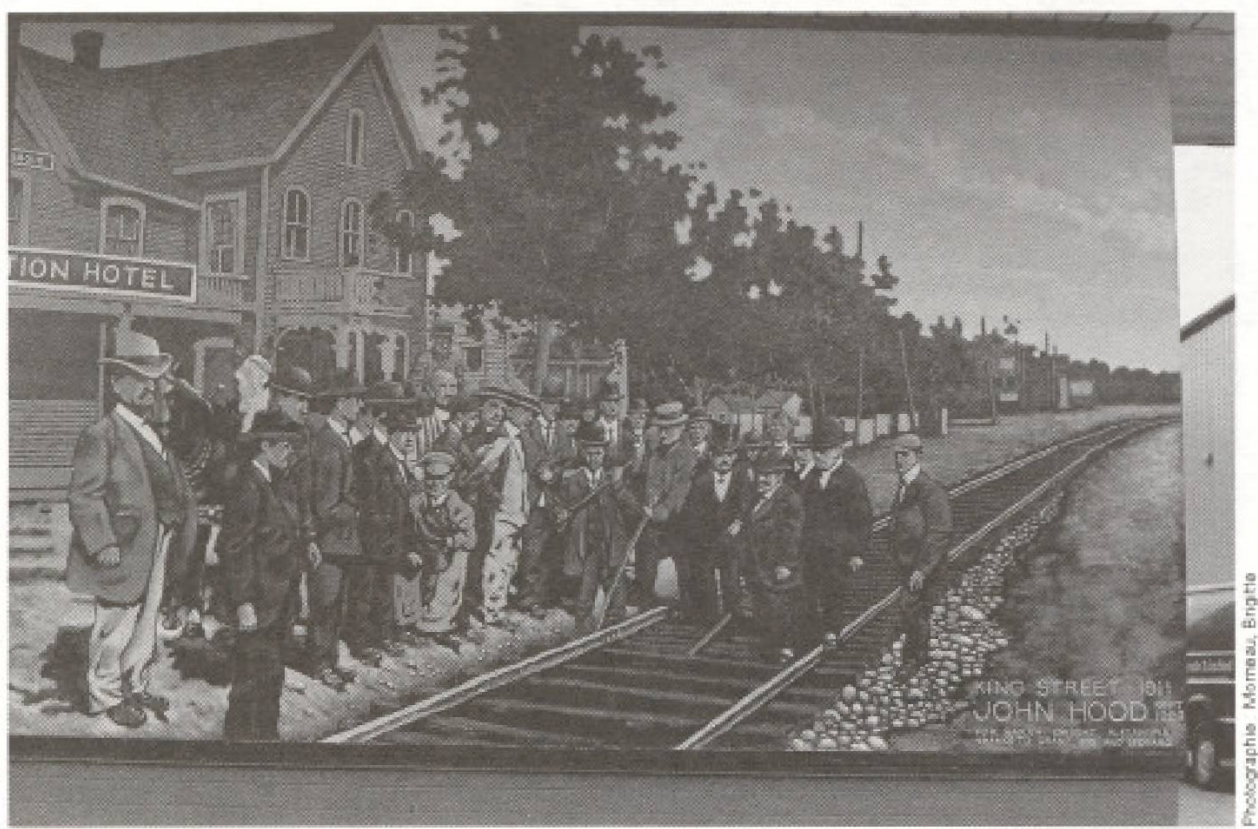

Photo no 4 : "THREE HISTORICAL SCENES" (1988), per John Hood, Welland, Ontario.

Celle murale complexe de $42 X 7^{\prime}$ esi en fait un fryptique qui représente en trois volets la vie socio-économique aux alentours de

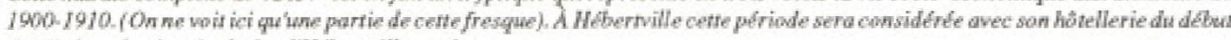
du siecle et le chemin de fer d'Heberwille station.

d'en mettre littéralement plein la vue aux visiteurs ${ }^{(14)}$,

Au-delà de ce projet particulier se pose aussi lanécessité d'aller plus loin dans le raisonnementet de considérerdenouvelles hypothèses de développement. Pour le moment, la tendance dominante est d'ériger presque partout des musces fermếs, des bibliothèques closes,
Or, le touriste semble rechercher en région une relation très sensitive et directe avec l'univers culturel local, ayant souvent, au préalable, visité des musées urbains aux collections prestigieuses. De plus, nos observations nous ont fait découvrir qu'effectivement les produits culturels out étaient plus en demande que les in quand il s'agissait de visiter des localités.

Ce constat nous amène alors à réfléchir sur les types d'aménagement à privilégier pour l'avenir. N'y aurait-il pas avantage à canaliser l'expression culturelle locale à des fins touristiques en la concentrant dans le grand espace régional extérieur à la manière des peuples primitifs, nomades, agraires ou marchands? Et ne serait-on pas à l'aubed'un nouveau type de réalisations culturelles régionales... complétant celles des cités!

Nous faisonsici référence au macro-art-landart-earthworks età l' art environnemental en général, qui, tous, tendent à marquer de culture humaine l'espace d'un champ, d'un désert, d'un lac, d' une falaise, d' un bâtiment, etc. D'où l'intérêt pour le touriste de se rendreen région pour y découvrir des produits nature/culture que lacité ne pourrait alors lui offrir. En un sens, nous sommes profondément convaincus que certaines régions sont dẹ́à mûres pour créer de nouveaux produits culturels et touristiques difficiles à réaliseren milieu urbain et rattachés au patrimoine lo$\mathrm{cal}$, sans imitation ni plagiat.

\section{Conclusion}

Malgré le fait que le projet muséal d'Hébertville s'achemine vers la réalisation d"une galerie d'art extérieure unique et capable, àelle seule, de créer une identité sousrégionale forte, ceci ne veut pas dire qu'il s'agit là d'une idée récente.

Le Guiness book of records situe la première murale en Turquie, 6200 ans avant J.-C.! A des époques encore plus reculées, il est aussi possible de se référer aux peintures rupestres extérieures faisant partie du patrimoine mondial de l'UNESCOet qui remontent à 25 000 années avant notre ère.

Près de nous, à Chemainus, en ColombieBritannique (Canada), un photographe du nom de Karl Schutz réactualisa le concept dès 1981. Ce qui entraîna par la suite la réalisation de presque trente murales et sculptures - la mise sur pied d'un festival mondial d'art populaire - ainsi... qu'un 
redéveloppement économique qui se traduisit, en huit ans, par l'implantation de plus de soixante-dix commerces, petites et moyennes entreprises touristiques. De quoi faire rêver plusieurs MRC ou municipalités en quête de projets originaux et rentables! ( voir photos no 2, 3 et 4)

L'étonnant dans tout cela, c'estque le Québec des régions $n$ "ait pas encore pu - pour des raisons qui nous echappent - profiter de ces nombreuses expériences réussies pour relancer ou ameliorer l'industrie culturelle et touristique à partir de ces bases très prometteuses. Le besoin est pourtant là, présent dans de nombreuses localités, pour freiner le dépeuplement et l'étiolement économique!

Malgré tout et quoique le projet d"Hébertville puisse essaimer en s'appliquant au milieu maritime nord-cottier-ou industriel nordique - ou encore - amérindien et anglophone tel que cela est déjà prévu, il n'en est pas moins vrai qu'il renferme des limites en termes de quantité et surtout.. de qualité

En ce sens, certains barbouillages abstraits ou grafitis contestataires muraux ne peuventêtre comparés au projetque nous venons de décrire! Il ne s"agit pas de croire à l'inspiration pour créer quelque part des ocuvres d'art populaires, qui deviendront des produits culturels et touristiques de valeur. La réussite en ce domaine exige une cohérence, des connaissances historiques précises, unemaîtrise artistiqueet des techniques particulières!

Mais, à part cette mise en garde, il faut reconnaître que l' artenvironnemental tel que partiellement abordé ici est si riche et performant, qu'il devrait permettre de doter plusieurs régions du Québec d'un produit et d'une image culturelle/touristique enrichie.

Enfin, en rédigeant cet article, nous avons voulu attirer l'attention sur le fait que la mondialisation des marches ne permet plus de s'en tenir à une vision trop conservatrice et traditionnelle du développement et des aménagements patrimoniaux et muscologiques. Il faudra de plus en plus étonner le touriste, l'émerveiller, le surprendre pour capter son attention et ainsi le faire venir et le retenir dans un endroit pour quelques heures ou quelques jours! A cela s'ajoute que le tourisme est un des trois secteurs économiques prometteur pour l'avenir et que cette industrie sera utile ou indispensable pour la relance de nombreuses localités et régions d'ici l'an 2000.

\section{NOTES ET RÉFÉRENCES}

11) Donnés foumies par la municipalité

(2) a... sacraliser veut gire investir d'importance, sus. citer le sens du stacré chez le fidele et le oratiquant [...] Méme si toutes les sacralisations ne visent. pas a jouer avec le sens du sacrd I. I, elles visent toujours a sortir un objet ou un lieu de l'ordinaire. de lianonymat, de la banalité. Elle opere done toujours une distinction.... . Laplante, Marc et Trottier, Louise, pp. $12-13$ et 22

(3) Un nombre important de maisons historiques comme Place-Poysle a Québecest un... «gisement patrimonials - 200000 dies sauvages a CapToumente est un... agisement fauniques, etc.

14) A considérer dans le sens de l'art figuratif ou naif, comme par exemple la maison Villeneuve à Chicoutimi.

(5) a... il est [... I facile de capterl'attention du touriste avec les ressources patrimoniales. On peut le faire d'autant plus que ces ressources se prétent facilement à des mises en scóne séduisantes: In montages habilesd'images d'un autretemps, etc. (ici les murales], Laplante, Marc et Trottier, Lou ise, p. 27.

(6) Discipline scientifique traitant de la biologie du comportement.

(7) Discipline scientifique tratant des distances entre les gens et les choses.

(8) Effectuéepar l'Universite Laval sous la formed'un laboratoire pratiqueen deuxième année de maltrise A.T.D.R.

(9) L'b́tude conceptuelle fut complétée par un plan de commercialisation effectué par la firme ÉCARTTANGRAM de Québec.

(10) Concu par la firme ECART-TANGRAM - finance par la C.F.P. et pédagogiquement acoompagné par le CÉGEP de St-Fúlicien.

(11) Cette agence appelee nvillage, vacances, familes d'Hébertuillew receura dejä, en 1992, une centaine d"autobus de touristes francas:

(12) Données foumies par la municipalité. Plus précisément, la municipalité s'est associée à une corporation a but non lucratif pour gerer l'ensemble des operations financieres.

(13) II s'agit la d'une prise de position fondamentale contenue dans le projet de nouvelle politique culturelle du Quebee.

(14] *... en donner à voir aux étrangers, en mettre plein la vue souvent, pour les impressionner * , Laplante: Marcet Trottier, Louise, p. 13

\section{BIBLIOGRAPHIE}

ATHENS, Circuit patrimonial et prospectus touristique: Home of the Historical Murals. 1992, $2 \mathrm{p}$ BERGEAON, GAGNON INC. Mise en valour du patimoine Habertvillois, rapport final, 1992, 95 p.

Chemainus, A Celebration of Canada Heritage: the Little Town That Did, Vancouver lstand, British Columbia, published by Chemainus of murals society, $1989,71 \mathrm{p}$.

GOUNEANEMENT DU QUEBEC, ministére des Affaires culturelles du Québec. La politique cultur elle du Outbec, 1992, $109 p$.

Hébert. Pierre-Maurice, Le curé Habart un sibcle d'histoire, 1810-1888, tome 1, Éditions de l'Écho. Montréal, 1968, $405 p$.

Jay-Payon, Jean-Claude et collaborateurs, Planification, conception ot localisation du produit récré. touristique d'Hobertville - P.C.L. Université Laval, ATDR, porte-folio carto-photographique ot document écrit, $1990,62 \mathrm{p}$.
Laplante, Marc et Louise Trottier, Concept d'interpretation du patrimoine de la ville de Qubbec las dimensions touristiques, pour le bureau d'études Cultura INC, $1968,41 \mathrm{p}$.

LE GROUPE-CONSEIL SUR LA POLITIQUE CUL. TUAELLE DU OUEBEC, Une politique de la culture ot des arts. sous la présidence de Roland Arpin, Les publications du Cuébec, 1991, $312 \mathrm{p}$.

UNESCO, L'artrupestreprehistorique: le patrimoine de Thumanite, Editions Bordas francophone $1991,32 \mathrm{p}$.

SÉGUIN, Normand, La conquàte du sol au 19eme siecle, Éditions du Boréal Express, 1977, 225 p. 